\title{
FOCAL SETS AND REAL HYPERSURFACES IN COMPLEX PROJECTIVE SPACE ${ }^{1}$
}

\author{
BY
}

\author{
THOMAS E. CECIL AND PATRICK J. RYAN
}

\begin{abstract}
Let $M$ be a real submanifold of $C P^{m}$, and let $J$ denote the complex structure. We begin by finding a formula for the location of the focal points of $M$ in terms of its second fundamental form. This takes a particularly tractable form when $M$ is a complex submanifold or a real hypersurface on which $J \xi$ is a principal vector for each unit normal $\xi$ to $M$. The rank of the focal map onto a sheet of the focal set of $M$ is also computed in terms of the second fundamental form. In the case of a real hypersurface on which $J \xi$ is principal with corresponding principal curvature $\mu$, if the map onto a sheet of the focal set corresponding to $\mu$ has constant rank, then that sheet is a complex submanifold over which $M$ is a tube of constant radius (Theorem 1). The other sheets of the focal set of such a hypersurface are given a real manifold structure in Theorem 2. These results are then employed as major tools in obtaining two classifications of real hypersurfaces in $C P^{m}$. First, there are no totally umbilic real hypersurfaces in $C P^{m}$, but we show:

THEOREM 3. Let $M$ be a connected real hypersurface in $C P^{m}, m>3$, with at most two distinct principal curvatures at each point. Then $M$ is an open subset of a geodesic hypersphere. Secondly, we show that there are no Einstein real hypersurfaces in $C P^{m}$ and characterize the geodesic hyperspheres and two other classes of hypersurfaces in terms of a slightly less stringent requirement on the Ricci tensor in Theorem 4.
\end{abstract}

One of the first results in the geometry of submanifolds is that an umbilic hypersurface $M$ in Euclidean space must be an open subset of a hyperplane or sphere. The proof goes as follows: assume that the shape operator is a scalar multiple of the identity, $A=\lambda I$, and use the Codazzi equation to show that $\lambda$ is constant. Then either $\lambda=0$, in which case $M$ lies on a hyperplane, or the focal points

$$
f_{\lambda}(x)=x+(1 / \lambda) \xi
$$

$\xi$ the unit normal, all coincide, and $M$ lies on the sphere of radius $1 / \lambda$ centered at the unique focal point.

This simple idea suggests a plan of attack for classifying hypersurfaces in terms of the nature of the principal curvatures. Under fairly general conditions, the set of focal points corresponding to a principal curvature $\lambda$ can be given a differentiable

Received by the editors December 19, 1980. Presented to the Society at its annual meeting in San Francisco, January 10, 1981.

1980 Mathematics Subject Classification. Primary 53B25, 53C40.

Key words and phrases. Focal sets, real hypersurfaces in complex projective space, umbilic hypersurfaces, Einstein hypersurfaces.

${ }^{1}$ Research partially supported by Summer Faculty Fellowships from the College of the Holy Cross and Indiana University. 
manifold structure with $f_{\lambda}$ collapsing each leaf of the corresponding principal distribution to a point [4]. This structure was used in an essential way in the classifications of taut hypersurfaces in [2] and [5].

In general, the study of hypersurfaces of real space forms has been a fertile field for differential geometers over the past decade. Much of the work has involved finding sufficient conditions for a hypersurface to be one of the "standard examples" (see [17, p. 251]), characterized by the fact that they have one or two distinct constant principal curvatures. The method of proof typically consists of algebraically determining the number of distinct principal curvatures, and then proving they are constant by use of the Codazzi equation and whatever algebraic relationships are available. Finally, one shows that the principal distributions are parallel, so that the de Rham theorem can be applied. In recent years, several authors have asked similar questions about real hypersurfaces in complex space forms. In this paper, we will develop the manifold structure of the focal set for submanifolds of $C P^{m}$, and exploit it to obtain some classifications of real hypersurfaces.

Real hypersurfaces in complex space forms come equipped with a distinguished tangent vector field $J \xi$ obtained by applying the complex structure $J$ to the unit normal field $\xi$. Whether or not $J \xi$ is a principal vector is of ten crucial in obtaining classifications, and at times it has been assumed in order to obtain the desired results. In Theorem 1, we have a geometric interpretation of the condition that $J \xi$ is principal, namely, the hypersurface $M$ must lie on a tube of constant radius over a complex submanifold of $C P^{m}$. This turns out to be a rather powerful tool, since it brings into play the more rigid structure of complex submanifolds.

Although there are no umbilic hypersurfaces in $C P^{m}$ (a fact first noted by Tashiro and Tachibana [22]), one sheet of the focal set of a geodesic hypersphere is precisely its center. We prove the following analogue of the classical theorem.

THEOREM 3. Let $M$ be a connected real hypersurface in $C P^{m}, m \geqslant 3$, with at most two distinct principal curvatures at each point. Then $M$ is an open subset of a geodesic hypersphere.

We first use the Codazzi equation to show that $J \xi$ is principal, and then use the fact that $M$ is a tube over a complex submanifold. A geodesic hypersphere in $C P^{m}$ has two distinct constant principal curvatures of respective multiplicities $2 m-2$ and 1, with $J \xi$ corresponding to the latter. R. Takagi [20] proved the conclusion of Theorem 3 under the assumption of two constant principal curvatures. His proof is quite different in spirit in that he first shows that the inverse image of $M$ under the projection $\pi: S^{2 m+1} \rightarrow C P^{m}$ is a hypersurface with two constant principal curvatures in the sphere $S^{2 m+1}$, and then invokes Cartan's classification of such hypersurfaces of the sphere to complete the proof.

A Riemannian manifold $M$ is Einstein if its Ricci tensor is a scalar multiple of the identity at each point. Einstein hypersurfaces of real space forms were classified through the work of Cartan and Thomas [23] and Fialkow [8], while Einstein complex hypersurfaces of complex space forms were classified by Smyth [19]. Two important classes of real hypersurfaces in $C P^{m}$ which arise in this context are the 
tubes of constant radius over a totally geodesic $C P^{k}, 0<k<m-1$, and tubes over a complex quadric $Q^{m-1}$. These were characterized by Takagi [21] as the only complete real hypersurfaces in $C P^{m}$ with three distinct constant principal curvatures, although the description of them as tubes is new in this paper. We show that there are no Einstein real hypersurfaces in $C P^{m}$ and characterize certain of these examples in terms of a slightly less stringent restriction on the Ricci tensor as follows. (Here $g$ is the Fubini-Study metric of constant holomorphic sectional curvature 4.)

THEOREM 4. Let $M$ be a connected real hypersurface in $C P^{m}, m \geqslant 3$, whose Ricci tensor $S$ satisfies the identity

$$
S X=a X+b g(X, J \xi) J \xi
$$

for some smooth functions $a$ and $b$ on $M$. Then $M$ is an open subset of one of the following:

(a) a geodesic hypersphere,

(b) a tube of radius $r$ over a totally geodesic $C P^{k}, 0<k<m-1$, where $0<r<\pi / 2$ and $\cot ^{2} r=k /(m-k-1)$,

(c) a tube of radius $r$ over a complex quadric $Q^{m-1}$ where $0<r<\pi / 4$ and $\cot ^{2} 2 r=m-2$.

The major part of the proof is to show that if $M$ satisfies this restriction on $S$, then $M$ is an open subset of a geodesic hypersphere, or a tube over some $C P^{k}$ or $Q^{m-1}$. A direct computation then shows that the hypersurfaces listed in (b) and (c) are the only such tubes which actually satisfy the requirement on the Ricci tensor.

Kon [10] proved Theorem 4 under the additional assumption that $a$ and $b$ are constant. Using that hypothesis, he shows that $M$ must have two or three constant principal curvatures and then invokes the results of Takagi. On the other hand, by also considering the structure of the focal set, we are able to obtain the more general result without appealing to Takagi's theorems. Finally, Maeda [11] also obtained partial results in the direction of Theorem 4. By use of Theorems 1 and 3, we are able to handle the cases which Maeda had left unresolved.

1. Preliminaries. We begin by recalling the construction of the Fubini-Study metric on $C P^{m}$ (see [9, Vol. II, pp. 273-278] and [13, pp. 514-515], for more detail). Let

$$
(z, w)=\sum_{k=0}^{m} z^{k} \bar{w}^{k}
$$

be the natural Hermitian inner product on $C^{m+1}$. The Euclidean metric on $C^{m+1}$ is given by $\langle z, w\rangle=\operatorname{Re}(z, w)$. The unit sphere $S^{2 m+1}$ in $C^{m+1}$ is a principal fibre bundle over $C P^{m}$ with structure group $S^{1}$ and projection map $\pi$. The tangent space to $S^{2 m+1}$ at a point $z$ is

$$
T_{z} S^{2 m+1}=\left\{w \in C^{m+1} \mid\langle z, w\rangle=0\right\} .
$$

Let

$$
T_{z}^{\prime}=\left\{w \in C^{m+1} \mid\langle z, w\rangle=\langle i z, w\rangle=0\right\}
$$


The distribution $T_{z}^{\prime}$ defines a connection in the principal fibre bundle $S^{2 m+1}\left(C P^{m}, S^{1}\right)$ in that $T_{z}^{\prime}$ is complementary to the subspace $\{i z\}$ tangent to the fibre through $z$, and $T_{z}^{\prime}$ is invariant by the action of $S^{1}$. The projection $\pi$ induces a linear isomorphism $\pi_{*}$ of $T_{z}^{\prime}$ onto $T_{\pi(z)} C P^{m}$. The Fubini-Study metic $g$ of constant holomorphic sectional curvature $c$ is defined by $g(X, Y)=(c / 4)\left\langle X^{\prime}, Y^{\prime}\right\rangle$, where $X, Y \in T_{p} C P^{m}$ and $X^{\prime}, Y^{\prime}$ are their respective horizontal lifts at $z$, where $\pi(z)=p$. For convenience, we will take $c=4$. The complex structure on $T^{\prime}$ defined by multiplication by $i$ induces the canonical complex structure $J$ on $C P^{m}$ through $\pi_{*}$.

Given a vector field $X$ on $C P^{m}$, there is a corresponding basic vector field $X^{\prime}$ on $S^{2 m+1}$ (in the terminology of O'Neill [14, p. 460]) such that at $z \in S^{2 m+1}, X_{z}^{\prime} \in T_{z}^{\prime}$ and $\left(\pi_{*}\right)_{z} X_{z}^{\prime}=X_{\pi(z)}$. If $X, Y$ are vector fields on $C P^{m}$, the Kählerian covariant derivative takes the form

$$
\tilde{\nabla}_{X} Y=\left(\pi_{*}\right) \nabla_{X^{\prime}}^{\prime} Y^{\prime}
$$

where $X^{\prime}, Y^{\prime}$ are the basic vector fields corresponding to $X, Y$ and $\nabla^{\prime}$ is the Levi-Civita connection on $S^{2 m+1}$.

2. Location of the focal points. For this local calculation, assume that $M$ is an embedded real $n$-dimensional $C^{\infty}$-submanifold of $C P^{m}$. Let $N M$ denote the normal bundle of $M$ with projection $P$ onto $M$. For $\xi \in N M$, let $F(\xi)$ be the point in $C P^{m}$ reached by traversing a distance $|\xi|$ along the geodesic in $C P^{m}$ originating at $x=P(\xi)$ with initial tangent vector $\xi$. A point $p \in C P^{m}$ is called $a$ focal point of multiplicity $\nu>0$ of $(M, x)$ if $p=F(\xi)$ and the Jacobian of $F$ has nullity $\nu$ at $\xi$.

Assume now that $\xi$ is a unit normal to $M$ at the point $x$. Let $w \in S^{2 m+1}$ with $\pi(w)=x$, and let $\xi^{\prime}$ be the horizontal lift of $\xi$ to $T_{w}^{\prime}$. The geodesic in $C P^{m}$ with initial tangent vector $\xi$ is given by

$$
\gamma(r)=\pi\left(\cos r w+\sin r \xi^{\prime}\right)
$$

Of course, this is independent of the choice of $w$. Noting that $\gamma(r)=\gamma(r+\pi)$ and by using $-\xi$ instead of $\xi$, if necessary, we need consider only $r \in[0, \pi / 2]$ in computing $\left(F_{*}\right)_{r \dot{\xi}}$. A simple computation shows that if $r=0$, then $F_{*}$ is the identity transformation, and so no focal point occurs. Thus we now assume $0<r \leqslant \pi / 2$.

Let $\left\{\xi_{1}, \ldots, \xi_{k}\right\}$ be an orthonormal basis for the normal space $T_{x}^{\perp} M$ with $\xi_{1}=\xi$. Let $U$ be a sufficiently small normal coordinate neighborhood of $x$, as defined in [9, Vol. I, p. 148]. Extend $\xi_{1}, \ldots, \xi_{k}$ to orthonormal normal vector fields on $U$ by parallel translation with respect to the normal connection $\nabla^{\perp}$ along geodesics in $M$. For any $u \in U, \eta \in T_{u}{ }^{\perp} M$, we can write

$$
\eta=\mu\left(\left(1-\sum_{j=2}^{k} t_{j}^{2}\right)^{1 / 2} \xi_{1}+t_{2} \xi_{2}+\ldots+t_{k} \xi_{k}\right)
$$

where $0 \leqslant \mu, 0 \leqslant\left|t_{j}\right| \leqslant 1$ for all $j$ and $\sum_{j=2}^{k} t_{j}^{2} \leqslant 1$. That is, $\mu=|\eta|$ and the $t_{j}$ are the direction cosines of $\eta$. The tangent space at this point $\eta$ in $N M$ can be considered as

$$
T_{u} M \times \operatorname{span}\left\{\partial / \partial \mu, \partial / \partial t_{2}, \ldots, \partial / \partial t_{k}\right\}
$$


We now begin to locate the focal points by computing $\left(F_{*}\right)_{r \xi}\left(\partial / \partial t_{j}\right)$. Let $z=\cos r w+\sin r \xi^{\prime}$ as before. Then $F(r \xi)=\pi(z)$ and $\left(F_{*}\right)_{r \xi}\left(\partial / \partial t_{j}\right)=\left(\pi_{*}\right)_{z} V$, where $V$ is the initial tangent vector to the curve,

$$
\eta(t)=\cos r w+\sin r\left(\cos t \xi_{1}^{\prime}+\sin t \xi_{j}^{\prime}\right),
$$

and $\xi_{1}^{\prime}, \xi_{j}^{\prime}$ are the respective horizontal lifts of $\xi_{1}(x), \xi_{j}(x)$ to $T_{w}^{\prime}$. One computes that $V=\sin r \xi_{j}^{\prime}$. To find the component of $V$ in $T_{z}^{\prime}$, one must subtract $\langle V, i z\rangle i z$ to get

$$
\left(F_{*}\right)_{r \xi}\left(\partial / \partial t_{j}\right)=\left(\pi_{*}\right)_{z}\left(\sin r\left(\xi_{j}^{\prime}-\sin r\left\langle\xi_{j}^{\prime}, i \xi^{\prime}\right\rangle i z\right)\right),
$$

where the vector on the right is in $T_{z}^{\prime}$.

A similar routine calculation shows that

$$
\left(F_{*}\right)_{r \xi}(\partial / \partial \mu)=\left(\pi_{*}\right)_{z}\left(-\sin r w+\cos r \xi^{\prime}\right)
$$

Let $X \in T_{x} M$, we now compute $\left(F_{*}\right)_{r \xi}(X, 0)$, where we are using the formulation in (1) for the tangent space to $N M$ at $r \xi$. Let $\beta(t)$ be a curve in $M$ with initial tangent vector $\vec{\beta}(0)=X$. Let $\alpha(t)$ be the horizontal lift of $\beta$ to $S^{2 m+1}$ with $\alpha(0)=w, \vec{\alpha}(0)=X_{w}^{\prime}$. Then $F\left(r \xi_{1}(\beta(t))\right)$ is the image under $\pi$ of the curve

$$
\eta(t)=\cos r \alpha(t)+\sin r \xi_{1}^{\prime}(\alpha(t)),
$$

and thus

$$
\left(F_{*}\right)_{r \xi}(X, 0)=\left(\pi_{*}\right)_{z}(\vec{\eta}(0)) .
$$

Considering $\eta(t)$ as a curve in $C^{m+1}$, we find

$$
\vec{\eta}(0)=\cos r X_{w}^{\prime}+\sin r D_{X_{w}^{\prime}} \xi_{1}^{\prime},
$$

where $D$ is the Euclidean covariant derivative in $C^{m+1}$. Since $\left\langle X_{w}^{\prime}, \xi_{1}^{\prime}\right\rangle=0$, we have $D_{X_{w}^{\prime}} \xi_{1}^{\prime}=\nabla_{X_{w}^{\prime}}^{\prime} \xi_{1}^{\prime}$. Moreover,

$$
\left(\pi_{*}\right)_{w}\left(\nabla_{X_{w}^{\prime}}^{\prime} \xi_{1}^{\prime}\right)=\tilde{\nabla}_{X} \xi_{1}
$$

since $\xi_{1}^{\prime}$ is a horizontal vector field and $X_{w}^{\prime} \in T_{w}^{\prime}$. By definition,

$$
\tilde{\nabla}_{X} \xi_{1}=-A_{\xi_{1}} X+\nabla_{X}^{\perp} \xi_{1} \text {. }
$$

Recall that $\nabla_{X}^{\perp} \xi_{1}=0$ by construction of the field $\xi_{1}$. Hence, if we let $\left(A_{\xi} X\right)_{w}^{\prime}$ denote the horizontal lift of $A_{\xi} X$ to $T_{w}^{\prime}$, we have

$$
\nabla_{X_{w}^{\prime}}^{\prime} \xi_{1}^{\prime}=-\left(A_{\xi} X\right)_{w}^{\prime}+\left\langle\nabla_{X_{w}^{\prime}}^{\prime} \xi_{1}^{\prime}, i w\right\rangle i w,
$$

where, of course, $\left(\pi_{*}\right)_{w}(i w)=0$. Differentiating the equation $\left\langle\xi_{1}^{\prime}(\alpha(t)), i \alpha(t)\right\rangle=0$ and evaluating at $t=0$, one obtains

$$
\left\langle\nabla_{X_{w}^{\prime}}^{\prime} \xi_{1}^{\prime}, i w\right\rangle=-\left\langle\xi^{\prime}, i X_{w}^{\prime}\right\rangle .
$$

Using (2)-(5) and noting that $\left\langle\xi^{\prime}, i X_{w}^{\prime}\right\rangle=-\left\langle X_{w}^{\prime}, i \xi^{\prime}\right\rangle$, we have

$$
\left(F_{*}\right)_{r \xi}(X, 0)=\left(\pi_{*}\right)_{z}\left(\cos r X_{w}^{\prime}-\sin r\left(\left(A_{\xi} X\right)_{w}^{\prime}-\left\langle X_{w}^{\prime}, i \xi^{\prime}\right\rangle i w\right)\right),
$$

where the vector on the right is not necessarily in $T_{z}^{\prime}$. In order to avoid an extremely cumbersome formula, we will not write the horizontal component of this vector now. We summarize the data necessary to locate the focal points as follows: 
Proposition 2.1. With the local trivialization of NM given above and for $0<r \leqslant$ $\pi / 2$,

(a) $\left(F_{*}\right)_{r \xi}\left(\partial / \partial t_{j}\right)=\left(\pi_{*}\right)_{z} \sin r\left(\xi_{j}^{\prime}-\sin r\left\langle\xi_{j}^{\prime}, i \xi^{\prime}\right\rangle i z\right)$.

(b) $\left(F_{*}\right)_{r \xi}(\partial / \partial \mu)=\left(\pi_{*}\right)_{z}\left(-\sin r w+\cos r \xi^{\prime}\right)$.

(c) $\left(F_{*}\right)_{r \xi}(X, 0)=\left(\pi_{*}\right)_{z}\left(\cos r X_{w}^{\prime}-\sin r\left(\left(A_{\xi} X\right)_{w}^{\prime}-\left\langle X_{w}^{\prime}, i \xi^{\prime}\right\rangle i w\right)\right)$,

where the vector on the right is in $T_{z}^{\prime}$ in cases (a) and (b), but not necessarily in (c).

It seems that one must make further restrictions on the submanifold $M$ to find a neat formula for locating the focal points. The two cases which we will treat are when $J \xi$ is normal (e.g. $M$ is a complex submanifold) or when $J \xi$ is a principal vector of $A_{\xi}$, as is the case in many important real hypersurfaces in $C P^{m}$.

In the case where $J \xi$ is normal, Proposition 2.1(a) reduces to a form which will be important for later considerations.

Proposition 2.2. Suppose $\xi_{2}=J \xi$ is normal to $M$ at $x$. Then

$$
\left(F_{*}\right)_{r \xi}\left(\partial / \partial t_{2}\right)=\left(\pi_{*}\right)_{z} \frac{1}{2}(\sin 2 r)\left(-\sin r i w+\cos r i \xi^{\prime}\right)
$$

where the vector on the right is in $T_{z}^{\prime}$.

The location of the focal points in this case can now be made explicit. Here $T_{\lambda}$ denotes the eigenspace corresponding to an eigenvalue $\lambda$ of $A_{\xi}$.

Proposition 2.3. Suppose $\xi_{2}=J \xi$ is normal to $M$ at $x$. Then

(a) $\left(F_{*}\right)_{r \xi}(X, 0)=0$ if $\lambda=\cot r$ is an eigenvalue of $A_{\xi}$ and $X \in T_{\lambda}$.

(b) For $r=\pi / 2,\left(F_{*}\right)_{r \xi}\left(\partial / \partial t_{2}\right)=0$.

(c) $\left(F_{*}\right)_{r \xi}(X, V) \neq 0$ except as determined by (a) and (b).

In the case where $J \xi$ is principal, Proposition 2.1(c) reduces to a formula which occurs sufficiently of ten that we state it explicitly below.

Proposition 2.4. Suppose $J \xi$ is an eigenvector of $A_{\xi}$ with corresponding eigenvalue $\mu$. Then

$$
\left(F_{*}\right)_{r \xi}(J \xi, 0)=\left(\pi_{*}\right)_{z}(\cos 2 r-\mu(\sin 2 r / 2))\left(-\sin r i w+\cos r i \xi^{\prime}\right),
$$

where the vector on the right is in $T_{z}^{\prime}$.

Thus the location of the focal points in this case is given by the following.

Proposition 2.5. Suppose $J \xi$ is an eigenvector of $A_{\xi}$ with corresponding eigenvalue $\mu$. Then

(a) $\left(F_{*}\right)_{r \xi}(X, 0)=0$ if $\lambda=\cot r$ is an eigenvalue of $A_{\xi}$ and $X$ is a vector in $T_{\lambda}$ orthogonal to $J \xi$.

(b) $\left(F_{*}\right)_{r \xi}(J \xi, 0)=0$ if $\mu=2 \cot 2 r$.

(c) $\left(F_{*}\right)_{r \xi}(X, V) \neq 0$ except as determined by (a) and (b).

Note that since $\cot 2(r-\pi / 2)=\cot 2 r$, there are two focal points at a distance $\pi / 2$ from each other corresponding to the principal curvature $\mu$ along each normal geodesic. 
3. Shape operators for tubes. In dealing with submanifolds of real space forms, one can give a natural manifold structure to the focal set of a hypersurface [4]. Reckziegel [16] noted that the key to obtaining an analogous structure for submanifolds of higher codimension is that the focal set of a tube of constant radius over a submanifold $M$ is the union of the focal set of $M$ with $M$ itself. One can then apply the results in codimension one to the tube.

We have computed the shape operator of a tube over a submanifold $M$ of $C P^{m}$. As one might expect, we have not found a natural diagonalizing basis of eigenvectors of $A_{\xi}$ except in the special cases where $J \xi$ is normal or $J \xi$ is principal. We briefly outline the computation below, and then state the results in the two cases.

Let $M$ be a real $n$-dimensional submanifold of $C P^{m}$ and let $B M$ denote the bundle of unit normal vectors to $M$. The tube of radius $r$ over $M$ is defined by the map $\phi_{r}: B M \rightarrow C P^{m}$ given by $\phi_{r}(\xi)=F(r \xi)$, for $F$ as in $\S 2$. For sufficiently small values of $r$ at least, $\phi_{r}$ determines a real hypersurface of $C P^{m}$.

In the case where $M$ is a real hypersurface, it is customary to consider $\phi_{r}$ : $M \rightarrow C P^{m}$. Let $\xi$ be a local field of unit normals, then $\phi_{r}(x)=F(r \xi(x))$. For values of $r$ such that $\phi_{r}$ is an immersion, $\phi_{r} M$ is called the parallel hypersurface at oriented distance $r$ from $M$. In the remainder of this section, the reader is asked to supply the appropriate changes of notation for the case of a real hypersurface.

We wish to compute the shape operator $A_{r}$ for $\phi_{r}$ at the point $\xi \in B M$. Using the same local trivialization of $N M$ as before, one has that $T_{\xi} B M$ is isomorphic to $T_{x} M \times \operatorname{span}\left\{\partial / \partial t_{2}, \ldots, \partial / \partial t_{k}\right\}$. The computations of $\left(\phi_{r}\right)_{*}\left(\partial / \partial t_{j}\right)$ and $\left(\phi_{r}\right)_{*}(X, 0)$ are identical with the calculations of $\left(F_{*}\right)_{r \xi}$ of the same vectors, and thus the results are given in Propositions 2.1, 2.2 and 2.4.

The vector $\eta=\left(\pi_{*}\right)_{z}\left(-\sin r w+\cos r \xi^{\prime}\right)$, which is tangent at $\pi(z)$ to the geodesic in $C P^{m}$ from $x$ in the direction $\xi$, is a unit normal to $\phi_{r}$ at $\pi(z)$. By definition, for $(X, V)$ in $T_{\xi} B M$, one has

$$
\left(\phi_{r}\right)_{*}\left(A_{r}(X, V)\right)=-\tilde{\nabla}_{\left(\phi_{r}\right)_{*}(X, V)} \eta
$$

where $\eta$ has been extended to a field of unit normals to $\phi_{r}$.

In the two special cases, we know $\left(\phi_{r}\right)_{*}(X, V)$ quite explicitly from $\$ 2$. The required computation is thus quite similar to those of the last section. Care must be taken in obtaining the correct basic vector fields on $S^{2 m+1}$ before differentiating, and at times the formulas of O'Neill [14, p. 461] are useful. We will merely state the results here.

Proposition 3.1. Suppose $\xi_{2}=J \xi$ is normal to $M$ at $x$. Let $X_{1}, \ldots, X_{n}$ be a basis of principal vectors of $A_{\xi}$ with corresponding principal curvatures $\lambda_{j}=\cot \theta_{j}, 0<\theta_{j}$ $<\pi$. Then the shape operator $A_{r}$ of the tube $\phi_{r}$ is given in terms of its principal vectors by

(a) $A_{r}\left(\partial / \partial t_{2}\right)=-2 \cot 2 r\left(\partial / \partial t_{2}\right)$.

(b) $A_{r}\left(\partial / \partial t_{j}\right)=-\cot r\left(\partial / \partial t_{j}\right), 3 \leqslant j \leqslant k$.

(c) $A_{r}\left(X_{j}, 0\right)=\cot \left(\theta_{j}-r\right)\left(X_{j}, 0\right), 1 \leqslant j \leqslant n$. 
Note that in this case, Proposition 2.2 states that $\left(\phi_{r}\right)_{*}\left(\partial / \partial t_{2}\right)$ is a multiple of $J \eta$ at $\pi(z)$, where $\eta$ is the unit normal to $\phi_{r} M$. Hence, we have the following result.

COROLlaRY 3.2. Let $V$ be a real hypersurface in $C P^{m}$ which lies on a tube of constant radius over a complex submanifold of $C P^{m}$. Let $\eta$ be a unit normal vector to $V$. Then $J \eta$ is a principal vector of the shape operator $A_{\eta}$.

This corollary enables us to locate the focal points of a complex submanifold $M$. Choose a value $r$ so that $V=\phi_{r} M$ is a hypersurface (this can always be done for local considerations); then $J \eta$ is principal on $V$. Thus the focal points of $V$ can be located by Proposition 2.5. Comparing this with the formula for locating the focal points of $M$ (Proposition 2.3), we find that study of the focal sets of real hypersurfaces contains complete information on focal sets of complex submanifolds as follows:

COROllaRY 3.3. Let $M$ be a complex submanifold of $C P^{m}$ and $r$ a real number such that $\phi_{r} M$ is a real hypersurface. The focal set of $\phi_{r} M$ consists of the union of the focal set of $M$ with $M$ itself.

Secondly, we turn to the case where $J \xi$ is a principal vector of $A_{\xi}$. Note that in this case, $\left(\phi_{r}\right)_{*} J \xi$ is a multiple of $J \eta$ (Proposition 2.4).

Proposition 3.4. Suppose $J \xi$ is an eigenvector of $A_{\xi}$ with corresponding eigenvalue $2 \cot 2 \theta, 0<\theta<\pi / 2$. Suppose $J \xi, X_{2}, \ldots, X_{n}$ is a basis of principal vectors of $A_{\xi}$ with $A_{\xi} X_{j}=\cot \theta_{j} X_{j}, 2 \leqslant j \leqslant n, 0<\theta_{j}<\pi$. Then the shape operator $A_{r}$ of the tube $\phi_{r}$ is given in terms of its principal vectors by

(a) $A_{r}\left(\partial / \partial t_{j}\right)=-\cot r\left(\partial / \partial t_{j}\right), 2 \leqslant j \leqslant k$.

(b) $A_{r}\left(X_{j}, 0\right)=\cot \left(\theta_{j}-r\right)\left(X_{j}, 0\right), 2 \leqslant j \leqslant n$.

(c) $A_{r}(J \xi, 0)=2 \cot (2(\theta-r))(J \xi, 0)$.

4. The manifold structure of the sheets of the focal set. The purpose of this section is to exhibit the components of the focal set of a real hypersurface $M$ in $C P^{m}$ as submanifolds themselves under certain restrictions. The first restriction, necessitated by the formula for locating focal points, is that $J \xi$ be a principal vector. Note that this is satisfied by any tube over a complex submanifold by Corollary 3.2.

The second restriction is that the principal curvature, corresponding to the sheet of the focal set in question, has constant multiplicity. This assumption was necessary even for submanifolds of real space forms; for without it, one cannot guarantee that the principal curvature itself (and hence the focal set) is differentiable.

Let $M$ be an orientable real hypersurface in $C P^{m}$ with field of unit normals $\xi$. Assume that $J \xi$ is a principal vector field with corresponding principal curvature function $\mu$. Maeda proved the following basic fact [11, p. 533].

Proposition 4.1 (MAEDA). If $J \xi$ is principal, then the corresponding principal curvature $\mu$ is locally constant.

Hence, we may write $\mu=2 \cot 2 r$ for some constant $0<r<\pi / 2$ when making local computations. Recall that for a real hypersurface with a field of unit normals 
$\xi$, we consider $\phi_{r}: M \rightarrow C P^{m}$ by $\phi_{r}(x)=F(r \xi(x))$. From Proposition 2.5(b), we see that there are two sets of focal points corresponding to $\mu$. One sheet is $\phi_{r} M$, the other is $\phi_{r-\pi / 2} M$, the set of points at a distance $\pi / 2-r$ from $M$ in the opposite direction. In the next theorem, we will refer only to $\phi_{r} M$ although everything applies equally well to $\phi_{r-\pi / 2}$.

From Proposition 2.5, we see that $\phi_{r}$ has constant rank $2 m-2$ on any open set $U$ where the other principal curvatures of $M$ do not assume the value cot $r$. If some $\lambda \equiv \cot r$ on an open set $U$, then $\phi_{r}$ will have constant rank $2 m-2-\nu$ where $\nu$ is the multiplicity of $\lambda$.

Assume now that $\phi_{r}$ has constant rank $q$ on $M$. A standard application of the inverse function theorem (see, for example, Boothby [1, pp. 70-80]) implies that for each point $x \in M$ there is a coordinate neighborhood $U$ of $x$ with local coordinates $u^{1}, \ldots, u^{2 m-1}$ with origin at $x$ such that $\phi_{r}$ is an embedding of the slice $u^{q+1}=\cdots=u^{2 m-1}=0$ into $C P^{m}$. In this way, $\phi_{r} M$ is a $q$-dimensional real submanifold in a neighborhood of $\phi_{r}(x)$. Moreover, the distribution $T_{0}(x)=\{X \in$ $\left.T_{x} M \mid\left(\phi_{r}\right)_{*} X=0\right\}$ is integrable with $(2 m-1-q)$-dimensional leaves on $M$. The following local result shows that $\phi_{r} M$ is locally a complex submanifold of $C P^{m}$. Hence, we have a local converse to Corollary 3.2 under the assumption that $\phi_{r}$ has constant rank. If we also assume that $M$ is compact, then we can give $\phi_{r} M$ a global structure as an immersed complex submanifold (see Theorem 1 (global version) below).

THEOREM 1 (LOCAL VERSION). Let $M$ be a connected, orientable real hypersurface of $C P^{m}$ on which $J \xi$ is a principal vector with corresponding constant principal curvature $\mu=2 \cot 2 r$. Suppose the map $\phi_{r}$ has constant rank $q$ on $M$. Then:

(a) $q$ is even and every point $x_{0} \in M$ has a neighborhood $U$ such that $\phi_{r} U$ is an embedded complex $(q / 2)$-dimensional submanifold of $C P^{m}$.

(b) For each point $x$ in such a neighborhood $U$, the leaf of the foliation $T_{0}$ through $x$ intersects $U$ in an open subset of a geodesic hypersphere in the totally geodesic $C P^{m-q / 2}$ orthogonal to $T_{p}\left(\phi_{r} U\right)$ at $p=\phi_{r}(x)$. Thus $U$ lies on the tube of radius $r$ over $\phi_{r} U$.

Proof. Given $x_{0}$, assume that $U$ is a sufficiently small neighborhood of $x_{0}$ in $M$ so that $V=\phi_{r} U$ is an embedded real $q$-dimensional submanifold, as given by the inverse function theorem. The proof of (a) is accomplished by showing that for each $p \in V$, the normal space $T_{p}^{\perp} V$ is invariant under $J$.

The crucial step is to construct a basis for $T_{p}^{\perp} V$ consisting entirely of vectors of the form

$$
\eta(x)=\left(\pi_{*}\right)_{z}\left(-\sin r w+\cos r \xi_{w}^{\prime}\right)
$$

where $x \in U, \xi$ is the unit normal to $M$ at $x$ and $w, z$ and $\xi^{\prime}$ are related to $x$ and $\xi$ as in \$3. (Note that different choices of $\xi \in T_{p}^{\perp} V$ will require different choices of $x \in \phi_{r}^{-1}(p)$.) It is then an easy application of the formulas in Propositions 2.1 and 2.4 to show that $J \xi=J \eta(x)$ is orthogonal to $\left(\phi_{r}\right)_{*}\left(T_{x} U\right)$ and thus to $T_{p} V$. 
To show that such a basis exists, we first observe that the mapping $\eta$ sends $U$ into the bundle $B V$ of unit normals to $V$. On the other hand, if we let $\psi_{r}$ : $B V \rightarrow C P^{m}$ denote the map onto the tube of radius $r$ over $V$, we see that $\psi_{r}(-\eta(x))=x$ for all $x \in U$. Consequently, $\eta$ is a diffeomorphism onto an open set $\eta(U)$ in $B V$. Furthermore, if $B_{p}$ denotes the fibre of $B V$ at $p, \eta(U) \cap B_{p}$ is open in $B_{p}$ and hence contains a basis for $T_{p}^{\perp} V$ as required.

We have thus shown that $T_{p}^{\perp} V$ is invariant by $J$. Since $V$ is an embedded submanifold, this is sufficient to make $V$ a complex submanifold with complex structure induced from $J$ (see, for example, [9, Vol. II, p. 171]).

The proof of (b) is now almost immediate. Given $x \in U$, let $y$ be any point on the leaf of $T_{0}$ through $x$. Since $\eta(y) \in B_{p}$ (as the previous construction shows), $y$ lies at a distance $r$ from $p$ along a geodesic of $C P^{m}$ normal to $\phi_{r} M$. Thus, the leaf is a submanifold of the geodesic hypersphere of center $p$ and radius $r$ in the totally geodesic $C P^{m-q / 2}$ determined by $T_{p}^{i}\left(\phi_{r} U\right)$. Since the leaf has the same dimension as the hypersphere, it is an open submanifold.

To obtain a global version of the theorem, we note the following. Since $C P^{m}$ is simply connected, a compact embedded real hypersurface $M$ must be orientable. Suppose $M$ also satisfies the remaining hypotheses of Theorem 1. Since the leaves of $T_{0}$ are (by definition) connected, it follows from Theorem 1(b) that each leaf is an open subset of a certain geodesic $(2 m-q-1)$-dimensional sphere in $C P^{m}$. Consequently, the foliation is regular in the sense of Palais [15]. This, in turn, implies that the leaves of $T_{0}$ are closed subsets of $M[15$, p. 18] and are thus compact. So, each leaf is, in fact, a geodesic $(2 m-q-1)$-dimensional sphere in $C P^{m}$. Further, it follows from Palais that the space of leaves $M / T_{0}$ is a Hausdorff real $q$-dimensional manifold and $\phi_{r}$ factors through an immersion $\sigma_{r}$ of $M / T_{0}$ into $C P^{m}$. Finally, $M / T_{0}$ is given a complex structure by pulling back the local complex structure on $\sigma_{r}\left(M / T_{0}\right)$ given by Theorem 1(a). Hence, we have

THEOREM 1 (GLOBAL VERSION). Let $M$ be a connected, compact real hypersurface embedded in $C P^{m}$ on which $J \xi$ is a principal vector with corresponding constant principal curvature $\mu=2 \cot 2 r$. Suppose the map $\phi_{r}$ has constant rank $q$ on $M$. Then $\phi_{r}$ factors through a holomorphic immersion of the complex $(q / 2)$-dimensional manifold $M / T_{0}$ into $C P^{m}$.

We now turn to the other sheets of the focal set in the case where the hypersurface lies on the tube of radius $r$ over a complex submanifold $\bar{M}$. Specifically, let $M=\phi_{r} U$ where $U$ is open in $B \bar{M}$. As noted in Corollary 3.3 , in handling this case, we are also giving a manifold structure to the sheets of the focal set of $\bar{M}$ as well.

Suppose $\bar{\lambda}=\cot \bar{\theta}$ is a principal curvature of constant multiplicity $\nu$ on $U$. Corresponding to $\bar{\lambda}$ is a principal curvature $\lambda=\cot (\bar{\theta}-r)$ of $M$ with the same multiplicity (Proposition 3.1). Recall that the principal curvatures of a complex submanifold $\bar{M}$ occur in pairs, i.e., if $\alpha$ is a principal curvature of $A_{\beta}$ ( $\beta$ normal to $\bar{M}$ ) with principal vector $X$, then $-\alpha$ is the principal curvature corresponding to the principal vector $J X$. Thus, if $\bar{\lambda}$ has constant multiplicity on $U$, either $\bar{\lambda}$ never equals 0 or $\bar{\lambda}$ is identically 0 . 
If $\bar{\lambda}$ is identically 0 , then $\lambda=\cot (\pi / 2-r)$ on $M$. The set of focal points determined by $\lambda$ is simply $\phi_{\pi / 2-r} M$ by Proposition 2.5, and this is one of the sheets determined by $\mu=-2 \cot 2 r$ (Proposition 3.1) discussed in Theorem 1 .

Therefore, we now assume that $\bar{\lambda}>0$ on $U$ with constant multiplicity $\nu$.

Proposition 4.2. Suppose the real hypersurface $M$ in $C P^{m}$ lies on the tube of radius $r$ over the complex submanifold $\bar{M}$; specifically, $M=\phi_{r} U$, where $U$ is open in $B \bar{M}$. Suppose $\lambda$ is a principal curvature of constant multiplicity $\nu>1$ on $M$ arising from a principal curvature function $\bar{\lambda}>0$ on $U$. Then:

(a) The $\nu$-dimensional distribution $T_{\lambda}$ is integrable.

(b) $\lambda$ is constant along the leaves of $T_{\lambda}$.

(c) The leaves of $T_{\lambda}$ are umbilic submanifolds of $\mathrm{CP}^{m}$.

Proof. This result is proven for hypersurfaces of real space forms (see [18] for (a) and (b) and [4] for (c)) by the use of the Codazzi equation

$$
\tilde{R}^{T}(X, Y) \xi=\left(\nabla_{Y} A\right) X-\left(\nabla_{X} A\right) Y,
$$

where the notation on the left means the tangential component of the submanifold of the Riemannian curvature tensor $\tilde{R}$ of the ambient space. For real space forms $\tilde{R}^{T}(X, Y) \xi$ is always zero. The proof for submanifolds of $C P^{m}$ is simply to show that under the hypotheses of the theorem the Codazzi equation reduces to the form it took in the real case.

Using the known Riemann curvature tensor for $C P^{m}$, the Codazzi equation for a real hypersurface $M$ reduces to (see, for example, [11, p. 531])

$$
\left(\nabla_{X} A\right) Y-\left(\nabla_{Y} A\right) X=g(X, W) \phi Y-g(Y, W) \phi X+2 g(X, \phi Y) W,
$$

where $W=-J \xi$ and $\phi$ is the restriction of $J$ to $M$ defined by

$$
J X=\phi X+g(X, W) \xi .
$$

To prove (a) and (b) suppose $X, Y$ are in $T_{\lambda}$. We must show that the right-hand side of (6) is zero. Since $X, Y$ are in $T_{\lambda}, g(X, W)=0=g(Y, W)$. Next, one deduces that $g(X, \phi Y)=0$ as follows. Suppose $X=\left(\phi_{r}\right)_{*}(\bar{X}, 0), Y=\left(\phi_{r}\right)_{*}(\bar{Y}, 0)$ for $\bar{X}, \bar{Y}$ in $T_{\bar{\lambda}} \bar{M}$, as in the notation of Proposition 3.4. Then $J \bar{Y}$ is in $T_{-\bar{\lambda}}$, and thus $g(\bar{X}, J \bar{Y})=0$. Then by Proposition 2.1(c) (with $\phi_{r}$ instead of $F$ ), $g(X, J Y)=0$. Thus, $g(X, \phi Y)$ also vanishes. One now applies the known proof [18, p. 371] for submanifolds of real space forms to complete the proof of (a) and (b).

To prove (c), one again shows that the Codazzi equation reduces to the Euclidean case. In [4], the proof that a leaf $V$ of $T_{\lambda}$ is umbilic is obtained by examining the Codazzi equation for $X \in T_{\lambda}, Y \in T_{\lambda}{ }^{\perp}$ and taking the $T_{\lambda^{-}}$ component of the whole equation. In the situation at hand, the $T_{\lambda}$-component of the left-hand side gives exactly what was present in the Euclidean situation. Hence, to complete the proof, one only needs to show that the $T_{\lambda}$-component of the right-hand side of (6) is zero for $X \in T_{\lambda}, Y \in T_{\lambda}^{\perp}$. Since $g(X, W)=0$ and $W \in T_{\lambda}^{\perp}$, the only term to check is $g(Y, W) \phi X$. But by the very same argument used above, $\phi X$ is orthogonal to $T_{\lambda}$, and the proof is finished.

REMARK 4.3. Chen and Ogiue [7] showed that an umbilic submanifold of $C P^{m}$ is either totally geodesic or is a totally real extrinsic sphere (umbilic with nonzero 
parallel mean curvature vector). Another result of Chen [6] then states that a $k$-dimensional extrinsic sphere in $C P^{m}$ must, in fact, lie in a totally geodesic $\mathbf{R} P^{k+1}$ as an extrinsic sphere. Consequently, the foliation $T_{\lambda}$ in Proposition 4.1 is easily seen to be regular in the sense of Palais [15], and therefore the space of leaves $M / T_{\lambda}$ is a (possibly non-Hausdorff) manifold. If one assumes that $M$ is complete, then the leaves must be compact, and $M / T_{\lambda}$ is Hausdorff.

Finally, we exhibit the sheet of the focal set corresponding to $\lambda$ as a real submanifold in $C P^{m}$. Suppose $\lambda=\cot \theta, 0<\theta<\pi$, on $M$. The focal point $f_{\lambda}(x)$ of $(M, x)$ corresponding to $\lambda$ is $\pi(z)$ with $z=\cos \theta(x) w+\sin \theta(x) \xi_{w}^{\prime}$, where $\pi(w)=x$ and $\xi_{w}^{\prime}$ is the horizontal lift of $\xi_{x}$ to $T_{w}^{\prime}$. We now compute $\left(f_{\lambda}\right)_{*} X$ for a principal vector $X \in T_{x} M$. Assume $A X=\rho X$ for a principal curvature $\rho$ of $M$ at $x$. Let $\beta(t)$ be a curve in $M$ with $\beta(0)=x$ and $\vec{\beta}(0)=X$. Let $\alpha(t)$ be the horizontal lift of $\beta(t)$ to $S^{2 m+1}$ with $\alpha(0)=w$, and let $\theta(t)$ denote $\theta(\beta(t))$. Then $f_{\lambda}(\beta(t))$ is the image under $\pi$ of the curve

$$
\gamma(t)=\cos \theta(t) \alpha(t)+\sin \theta(t) \xi^{\prime}(\alpha(t)),
$$

and $\left(f_{\lambda}\right)_{*} X=\left(\pi_{*}\right)_{z} V$, where $V$ is the initial tangent vector to $\gamma(t)$. A computation very similar to that leading to Proposition 2.1(c) (except with $\theta$ variable) yields

$$
V=(X \theta) \eta+(\cos \theta(x)-\rho \sin \theta(x)) X_{w}^{\prime}-\sin \theta(x)\left\langle X_{w}^{\prime}, i \xi_{w}^{\prime}\right\rangle i w,
$$

where $\eta=-\sin \theta(x) w+\cos \theta(x) \xi_{w}^{\prime}$. Note that $\eta \in T_{z}^{\prime}$. Since $\lambda=\cot \theta$, one computes $X \lambda=-\left(1+\lambda^{2}\right) X \theta$. If $g(X, J \xi)=0$, then $X_{w}^{\prime} \in T_{z}^{\prime}$, and from (7) we obtain

$$
\left(f_{\lambda}\right)_{*} X=\left(\pi_{*}\right)_{z}\left(-(X \lambda) \eta /\left(1+\lambda^{2}\right)+(\cos \theta(x)-\rho \sin \theta(x)) X_{w}^{\prime}\right),
$$

where the vector on the right is in $T_{z}^{\prime}$. If $X=J \xi$, the right-hand side of (7) reduces as in Proposition 2.4, and we have

$$
\left(f_{\lambda}\right)_{*}(J \xi)=\left(\pi_{*}\right)_{z}\left(-J \xi(\lambda) \eta /\left(1+\lambda^{2}\right)+\left(\cos 2 \theta(x)-\frac{\mu}{2} \sin 2 \theta(x)\right) i \eta\right),
$$

where the vector on the right is in $T_{z}^{\prime}$, and we have written $\mu$ instead of $\rho$ for the principal curvature corresponding to $J \xi$.

From these formulas, we will now deduce the following result giving a manifold structure to the sheet of the focal set $f_{\lambda}(M)$.

THEOREM 2. Suppose the real hypersurface $M$ in $C P^{m}$ lies on the tube of radius $r$ over the complex submanifold $\bar{M}$; specifically, $M=\phi_{r} U$, where $U$ is open in $B \bar{M}$. Suppose $\lambda$ is a principal curvature of constant multiplicity $\nu$ on $M$ arising from a principal curvature function $\bar{\lambda}>0$ on $U$.

(a) If $\nu=1$ and $X \lambda \neq 0$ on $M$ for some vector field $X \in T_{\lambda}$, then $f_{\lambda}: M \rightarrow C P^{m}$ is an immersion.

(b) If $\nu=1$ and $X \lambda=0$ on $M$ for all $X \in T_{\lambda}$, then $f_{\lambda}$ factors through an immersion of the $(2 m-2)$-dimensional space of leaves $M / T_{\lambda}$.

(c) If $\nu>1$, then $f_{\lambda}$ factors through an immersion of the $(2 m-1-\nu)$-dimensional space of leaves $M / T_{\lambda}$.

Proof. (a) For a unit vector $X \in T_{\lambda}$ with $X \lambda \neq 0$, since $\cos \theta(x)-\lambda \sin \theta(x)=$ 0 , (8) becomes

$$
\left(f_{\lambda}\right)_{*} X=\left(\pi_{*}\right)_{z}(-X \lambda) \eta /\left(1+\lambda^{2}\right)
$$


and $\left(\pi_{*}\right)_{z} \eta$ is in the range of $\left(f_{\lambda}\right)_{*}$. For a principal vector $Y \in T_{\lambda}^{\perp}$ and orthogonal to $J \xi$, we see that by subtracting a suitable multiple of $\left(\pi_{*}\right)_{z} \eta$ from $\left(f_{\lambda}\right)_{*} Y$ in (8) that $\left(\pi_{*}\right)_{z} Y_{w}^{\prime}$ is in the range of $\left(f_{\lambda}\right)_{*}$. Similarly, in reference to (9), by recalling that $\mu=-2 \cot 2 r$, we get $\cos 2 \theta(x)-(\mu / 2) \sin 2 \theta(x) \neq 0$ from the assumption that $\bar{\lambda}>0$ on $U$. Again, by subtracting a multiple of $\left(\pi_{*}\right)_{z} \eta$, we have that $\left(\pi_{*}\right)_{z}(i \eta)$ is in the range of $\left(f_{\lambda}\right)_{*}$. The range of $\left(f_{\lambda}\right)_{*}$ is thus the $(2 m-1)$-dimensional space which is the image under $\left(\pi_{*}\right)_{z}$ of the space spanned by $\eta, i \eta$, and the set of vectors $Y_{w}^{\prime}$, where $Y$ is a principal vector orthogonal to $T_{\lambda}$ and to $J \xi$.

(b) and (c) Since $\eta$ is orthogonal to in and to $Y_{w}^{\prime}$ such that $Y \in T_{\lambda}^{\perp}$ and $g(J \xi, Y)=0$, we see from (8) and (9) that $\left(f_{\lambda}\right)_{*}$ is injective on the $(2 m-1-\nu)$ dimensional subspace $T_{\lambda}{ }^{\perp}$ at each $x \in M$. On the other hand, using $X \lambda=0$ for all $X \in T_{\lambda}$ (which follows from Proposition 4.2 in case (c)), we see from (8) that $\left(f_{\lambda}\right)_{*} \equiv 0$ on $T_{\lambda}$. Thus $\left(f_{\lambda}\right)_{*}$ has constant rank $2 m-1-\nu$, and by a basic result of Palais [15, p. 25], $f_{\lambda}$ factors through an immersion of the space of leaves $M / T_{\lambda}$ of the regular foliation $T_{\lambda}$.

EXAMPLES. 1. Geodesic hypersphere. Let $M$ be the set of points at a fixed distance $r<\pi / 2$ from a point $p \in C P^{m}$. By considering $M$ as a tube of radius $r$ over the 0 -dimensional manifold $\{p\}$, one computes from Proposition 3.1 that $M$ has two constant principal curvatures: $\lambda=\cot r$ of multiplicity $2 m-2$ and $\mu=2 \cot 2 r$ of multiplicity 1 at each point (with the proper choice of unit normal to $M$, of course). Alternatively, let $C P^{m-1}$ be the dual hyperplane at distance $\pi / 2$ from $p$. It is geometrically obvious that $M$ is also the tube of radius $\pi / 2-r$ over $C P^{m-1}$, and the principal curvatures of $M$ can likewise be computed using this fact. From Proposition 2.5, one finds that the focal set of $M$ consists of $\{p\} \cup C P^{m-1}$. The point $p$ is naturally a focal point of multiplicity $2 m-1$ of $(M, x)$ for each $x \in M$. On the other hand, for each $q \in C P^{m-1}$, there is a circle of radius $\pi / 2-r$ of points in $M$ having $q$ as a focal point of multiplicity one. This circle is a leaf of the foliation $T_{\mu}=\operatorname{span}\{J \xi\}$, and it is the intersection of $M$ with the totally geodesic $C P^{1}$ through $q$ orthogonal to $C P^{m-1}$ at $q$.

2. Tube over totally geodesic $C P^{k}(1 \leqslant k \leqslant m-2)$. For an integer $k, 1 \leqslant k \leqslant m$ -2 , and for $0<r<\pi / 2$, let $M^{\prime}(2 m, k, r)$ in $S^{2 m+1}$ be defined by

$$
\sum_{j=0}^{k}\left|z_{j}\right|^{2}=\cos ^{2} r, \quad \sum_{j=k+1}^{m}\left|z_{j}\right|^{2}=\sin ^{2} r .
$$

$M^{\prime}(2 m, k, r)$ is a standard product $S^{2 k+1} \times S^{2 l+1}, l=m-k-1$. The projection $\pi$ submerses $M^{\prime}(2 m, k, r)$ onto a real hypersurface in $C P^{m}$ which we denote $M(2 m-1, k, r)$. Substituting $r=0$ in (10), one obtains a totally geodesic $S^{2 k+1}$ in $S^{2 m+1}$ whose image under $\pi$ is a totally geodesic $C P^{k}$. One can easily check that $M(2 m-1, k, r)$ is the tube of radius $r$ over $C P^{k}$. From Proposition 3.1, one sees that $M(2 m-1, k, r)$ has three constant principal curvatures: $\lambda_{1}=\cot r, \lambda_{2}=$ $\cot (r-\pi / 2)$ and $\mu=2 \cot 2 r$ with respective multiplicities $2 l, 2 k, 1$. As in the first example, $M(2 m-1, k, r)$ is also a tube of radius $\pi / 2-r$ over the totally geodesic $C P^{l}$ obtained by substituting $\pi / 2$ into (10) and projecting into $C P^{m}$. (In fact, Example 1 is obtained by this construction with $k=0$.) The focal set of $M(2 m-1, k, r)$ is $C P^{k} \cup C P^{\prime}$. The foliation $T_{0}$ for the focal map $\phi_{r}$ is $T_{\lambda_{1}} \oplus T_{\mu}$, 
and for $\phi_{r-\pi / 2}, T_{0}=T_{\lambda_{2}} \oplus T_{\mu}$. In each case, the leaves of $T_{0}$ are geodesic spheres of the appropriate dimensions.

3. Tube over totally geodesic $\mathbf{R} P^{m}$ and over complex quadric $Q^{m-1}$. Let $V^{\prime}(2 m, r)$ be the real hypersurface in $S^{2 m+1}$ given by the equation

$$
\left|\sum_{j=0}^{m} z_{j}^{2}\right|^{2}=\cos ^{2} 2 r, \quad 0<r<\frac{\pi}{4} .
$$

As Nomizu [12, p. 197] demonstrates, $V^{\prime}(2 m, r)$ has four distinct constant principal curvatures. The projection $\pi$ submerses $V^{\prime}(2 m, r)$ onto a real hypersurface in $C P^{m}$ which we denote $V(2 m-1, r)$. Setting $r=0$ in (11), one obtains a solution set $\Omega=\left\{e^{i \theta} z \mid z_{j}\right.$ is real, $\left.0 \leqslant j \leqslant m\right\}$, and $\pi(\Omega)$ is well known to be a totally geodesic $\mathbf{R} P^{m}$ in $C P^{m} . V(2 m-1, r)$ is the tube of radius $r$ over $\mathbf{R} P^{m}$. For every normal $\eta$ to $\mathbf{R} P^{m}, J \eta$ is a principal vector of $A_{\eta}$. Hence by Proposition 3.4, one finds that $V(2 m-1, r)$ has three distinct constant principal curvatures: $\lambda_{1}=-\cot r$ and $\lambda_{2}=\cot (\pi / 2-r)$, both of multiplicity $m-1$, and $\mu=2 \cot 2(\pi / 4-r)$ of multiplicity 1 , whose principal vector is $J \xi$, where $\xi$ is the unit normal to $V(2 m-1, r)$. Clearly $\mathbf{R} P^{m}$ is the focal set of $V(2 m-1, r)$ corresponding to $\lambda_{1}$, and the leaves of $T_{\lambda_{1}}$ are $(m-1)$-dimensional extrinsic spheres. Note that when one traverses a distance $\pi / 2$ along a normal geodesic to $\mathbf{R} P^{m}$, one reaches another point of $\mathbf{R} P^{m}$. Thus $\mathbf{R} P^{m}$ is also the sheet of the focal set corresponding to $\lambda_{2}=\cot (\pi / 2-r)$. Finally, by setting $r=\pi / 4$ in (11), one obtains the equation $z_{0}^{2}+\cdots+z_{m}^{2}=0$ whose image under $\pi$ is the complex quadric $Q^{m-1}$. For a fixed $V(2 m-1, r)$, $0<r<\pi / 4$, the two focal points along each normal geodesic determined by $\mu$ are at a distance $\pi / 2$ apart, and they both lie on $Q^{m-1}$. Hence $V(2 m-1, r)$ is a tube of radius $\pi / 4-r$ over $Q^{m-1}$ and also a tube of radius $\pi / 4+r$ over $Q^{m-1}$. Note that we have also shown that the focal set of $Q^{m-1}$ is $\mathbf{R} P^{m}$, as was first pointed out by Cecil [3, p. 28]. Finally, by taking $t=\pi / 4-r$, our formulas for the principal curvatures can be made to agree with those of Takagi's example B [20, p. 47].

REMARK 4.3. Takagi showed that the geodesic hypersphere is the only real hypersurface in $C P^{m}$ with two constant principal curvatures [20], and that the $M(2 m-1, k, r)$ and $V(2 m-1, r)$ of Examples 2 and 3 are the only real hypersurfaces with three distinct constant principal curvatures [21]. Moreover, $J \xi$ is principal in all of the examples of homogeneous real hypersurfaces in $C P^{m}$ with constant principal curvatures in Takagi [20, p. 47]. Thus, these are all tubes over complex submanifolds. Weinstein [24] showed that certain geodesic hyperspheres in $C P^{m}$ are Berger spheres which provide counterexamples to the extension of Klingenberg's result on the length of closed geodesics to odd-dimensional manifolds. Little seems to be known about the Riemannian geometry of Examples 2 and 3 as well as the other examples of Takagi, and a study of them from this point of view would seem to be worthwhile.

5. Classifications of real hypersurfaces. Let $M$ be an embedded real hypersurface in $C P^{m}$. Let $\xi$ be a local field of unit normals to $M$ and let $W=-J \xi$. As noted earlier, the Codazzi equation has the form

$$
\left(\nabla_{X} A\right) Y-\left(\nabla_{Y} A\right) X=g(X, W) \phi Y-g(Y, W) \phi X+2 g(X, \phi Y) W,
$$


where $J X=\phi X+g(X, W) \xi$. We first prove

THEOREM 3. Let $M$ be a connected real hypersurface in $C P^{m}, m \geqslant 3$, with at most two distinct principal curvatures at each point. Then $M$ is an open subset of a geodesic hypersphere.

The proof is broken into three steps, and we separate the first two off as propositions.

Proposition 5.1 (TASHiro and Tachibana [22]). There is no umbilic real hypersurface in $C P^{m}$.

Proof. Suppose $A X=\lambda X$ for all $X$ tangent to $M$. Take $X$ and $Y$ orthogonal to $W$ and note that the Codazzi equation reduces to $(X \lambda) Y-(Y \lambda) X=2 g(X, \phi Y) W$. Thus $g(X, \phi Y)=0$ for all $X, Y$ orthogonal to $W$, which is contradicted when $X=\phi Y$.

The following is the key step in proving Theorem 3.

Proposition 5.2. Suppose a real hypersurface $M$ in $C P^{m}, m \geqslant 3$, has exactly two distinct principal curvatures at each point. Then $J \xi$ is a principal vector.

Proof. Let $\lambda$ and $\mu$ denote the two principal curvature functions, and suppose

$$
J \xi=a X+b V
$$

for nonzero functions $a$ and $b$, where $X \in T_{\lambda}$ and $V \in T_{\mu}$ are unit vector fields on some open subset of $M$ where $J \xi$ is not principal. Since dimension $M \geqslant 5$, at least one of $T_{\lambda}$ and $T_{\mu}$ has dimension at least 3, say $T_{\lambda}$. Let

$$
\Sigma=\left\{Y \in T_{\lambda} \mid g(Y, X)=0\right\}, \quad \Omega=\left\{Z \in T_{\mu} \mid g(Z, V)=0\right\} .
$$

Choose $Y, Z$ mutually orthonormal in $\Sigma$. Then from (12) we see that $Y$ and $Z$ are orthogonal to $W=-J \xi$, and the Codazzi equation becomes

$$
\left(\nabla_{Z} A\right) Y-\left(\nabla_{Y} A\right) Z=2 g(Z, \phi Y) W .
$$

Some standard algebra further reduces this to

$$
(Z \lambda) Y-(Y \lambda) Z+(\lambda I-A)[Z, Y]=2 g(Z, \phi Y) W .
$$

Since $W$ and $(\lambda I-A)[Z, Y]$ are orthogonal to $Y$ and $Z$, we conclude that $Y \lambda=0$ for all $Y \in \Sigma$. Moreover, the left-hand side then has no $T_{\lambda}$-component; this forces $g(Z, \phi Y)=0$. Therefore, $\phi Y$ is orthogonal to $\Sigma$. Now consider the Codazzi equation for $Y$ in $\Sigma$ and the particular vector field $X$. Since $X, Y$ are in $T_{\lambda}$ and $g(X, W)=$ $-a$, this reduces to

$$
(X \lambda) Y-(Y \lambda) X+(\lambda I-A)[X, Y]=-a \phi Y+2 g(X, \phi Y) W .
$$

We have shown that $Y \lambda=0$, and we now conclude that $X \lambda=0$, since all the other terms are orthogonal to $\Sigma$. Thus

$$
(\lambda I-A)[X, Y]=-a \phi Y+2 g(X, \phi Y) W .
$$

The left side is orthogonal to $X \in T_{\lambda}$, so taking the inner product with $X$ and recalling $g(X, W)=-a$, we obtain $-3 \operatorname{ag}(X, \phi Y)=0$. Since $\phi Y$ is orthogonal to $\Sigma$ and to $X$, we conclude $\phi Y \in T_{\mu}$. However, $g(\phi Y, a X+b V)=g(J Y, J \xi)=0$. 
Thus $b g(\phi Y, V)=0$, and $\phi Y$ is orthogonal to $V$. We have thus shown that $\phi(\Sigma) \subset \Omega$. Since $\phi Y=J Y$ for all $Y \in \Sigma, \phi$ is injective on $\Sigma$. Thus dimension $\Omega \geqslant \operatorname{dimension} \Sigma \geqslant 3$. Consequently, we may reverse the roles of $\lambda$ and $\mu$ above to show that $\phi(\Omega) \subset \Sigma$. Hence, $\Sigma$ and $\Omega$ have the same dimension, and taking into account $X$ and $V$, we have dimension $M=2($ dimension $\Sigma)+2$, i.e. $M$ is even dimensional. This is a contradiction, and we conclude that $a$ or $b$ is identically zero, and $J \xi$ is principal.

REMARK. This result and proof hold equally well in complex hyperbolic space, the complex space form with constant holomorphic sectional curvature -1 .

Proof of Theorem 3. Let $x$ be any nonumbilic. Fix a choice of unit normal $\xi$ near $x$ and write $\mu=2 \cot 2 r, 0<r<\pi / 2$, for the principal curvature with eigenvector $J \xi$. If the other principal curvature $\lambda$ is not equal to $\cot r$ at $x$, then $\phi_{r}$ has rank $2 m-2$ at $x$ (Propositions 2.1 and 2.4). Otherwise, $\phi_{r}$ has rank 0 there.

If $\phi_{r}$ has rank $2 m-2$ at $x$, the same condition holds in some umbilic-free neighborhood $U$ of $x$. By the local version of Theorem 1, $U$ may be chosen so that $N=\phi_{r} U$ is an embedded complex submanifold of complex codimension 1 in $C P^{m}$. Moreover, $U$ lies on a tube of radius $r$ over $N$. The formulas for the shape operators of such tubes (Proposition 3.1) yield that all eigenvalues of each shape operator of $N$ must be equal. But for complex submanifolds, the eigenvalues occur in pairs which are negatives of each other, and hence they are all zero in this case. This makes $N$ totally geodesic, an open subset of a complex projective hyperplane in $C P^{m}$. According to Proposition 3.1 the principal curvatures of $U$ are $-2 \cot 2 r$ $=\cot (2(\pi / 2-r))$ and $\cot (\pi / 2-r)$ or, by reversing the normal, $\mu=2 \cot 2 r$, $\lambda=\cot (r-\pi / 2)$. Using the fact that the principal curvatures are locally constant near nonumbilics where $\phi_{r}$ has rank $2 m-2$, one can show that they are also locally constant near nonumbilics where $\phi_{r}$ has rank 0 simply by considering $\phi_{r-\pi / 2}$. From this, one shows rather easily that every point of $M$ is nonumbilic. This allows $\xi$ to be defined globally, say by insisting that the principal curvature $\lambda$ of multiplicity $2 m-2$ be positive (it does not equal zero). Thus, we can write $\lambda=\cot t, 0<t<\pi / 2$, where $t$ is either the value $r$ mentioned above or $\pi / 2-r$. In either case, $\mu=2 \cot 2 t$, and $\phi_{t}$ has rank 0 on all of $M$. Hence, $\phi_{t} M$ is a single point, and $M$ is an open subset of a geodesic hypersphere centered at this point.

Finally, we turn to the question of real Einstein hypersurfaces in $C P^{m}$. Suppose that the Ricci tensor $S$ of $M$ satisfies

$$
S X=a X+b g(X, W) W
$$

for some smooth functions $a$ and $b$ on $M$. Of course, if $b=0$, then $M$ is Einstein. We will show that (13) implies that $M$ is an open subset of one of the examples of $\$ 4$ with 2 or 3 constant principal curvatures. A straightforward calculation shows that $b \neq 0$ for these examples, and hence there are no Einstein real hypersurfaces in $C P^{m}$.

From the Gauss equation, one computes (see, for example, [11, p. 532]) that the Ricci tensor of an arbitrary real hypersurface in $C P^{m}$ has the form

$$
S X=(2 m+1) X-3 g(X, W) W+h A X-A^{2} X,
$$


where $h=\operatorname{trace} A$. If (13) is also satisfied, one obtains

$$
\left(A^{2}-h A+\rho\right) X=\sigma g(X, W) W,
$$

where the functions $\rho=a-2 m-1$ and $\sigma=-(3+b)$. The next proposition was first proven by Maeda [11, p. 536].

PROPOSITION 5.3. If $\sigma \neq 0$ on an open set $U$, then $J \xi$ is principal, and there are at most three distinct principal curvatures at each point of $U$.

Proof. Let $K$ be the symmetric tensor $A^{2}-h A$. From (14) we see that $K W=$ $(\sigma-\rho) W$, so $W$ is an eigenvector of $K$. If $X$ is any eigenvector of $K$ orthogonal to $W$, $K X=-\rho X$. Hence if $\sigma \neq 0, \sigma-\rho$ is an eigenvalue of multiplicity one of $K$. On the other hand, if $X$ is an eigenvector of $A$, then clearly $X$ is an eigenvector of $K$. If $\left\{X_{1}, \ldots, X_{2 m-1}\right\}$ is an orthonormal basis of eigenvectors of $A$, then it is also a basis of eigenvectors for $K$. There must be a unique $X_{i}$ so that $K X_{i}=(\sigma-\rho) X_{i}$, and so $X_{i}= \pm W$, and $W$ is an eigenvector for $A$.

Let $A_{0}$ be the restriction of $A$ to the orthogonal complement $W^{\perp}$ of $W$. Since $W$ is principal, $A_{0}$ leaves $W^{\perp}$ invariant and, by (14), $A_{0}^{2}-h A_{0}+\rho=0$. Thus $A_{0}$ has at most two distinct eigenvalues, and $M$ has at most three distinct principal curvatures.

In the case $\sigma=0(b=-3)$, one cannot use the same argument to show that $J \xi$ is principal, and this case was excluded by Maeda. However, we do have

Proposition 5.4. If $\sigma=0$ on an open set $U$, then there exist at most two distinct principal curvatures at each point of $U$.

Proof. With $\sigma=0,(14)$ becomes $\left(A^{2}-h A+\rho\right) X=0$ for all $X$ tangent to $M$. Thus at most two eigenvalues of $A$ can be distinct.

We will now invoke Theorem 3, which Maeda did not have, to show that when $\sigma=0, U$ is an open subset of a geodesic hypersphere. This leads to a proof of

THEOREM 4. Let $M$ be a connected real hypersurface in $C P^{m}, m \geqslant 3$, whose Ricci tensor $S$ satisfies the identity $S X=a X+b g(X, J \xi) J \xi$ for some smooth functions $a$ and $b$ on $M$. Then $M$ is an open subset of one of the following:

(a) a geodesic hypersphere,

(b) a tube of radius $r$ over a totally geodesic $C P^{k}, 0<k<m-1$, where $0<r<\pi / 2$ and $\cot ^{2} r=k /(m-k-1)$,

(c) a tube of radius $r$ over a complex quadric $Q^{m-1}$ where $0<r<\pi / 4$ and $\cot ^{2} 2 r=m-2$.

Proof. By Propositions 5.3 and 5.4, there exist at most three distinct principal curvatures at each point of $M$. If there are less than three distinct principal curvatures at every point, then $M$ is an open subset of a geodesic hypersphere by Theorem 3. If not, the set $\Sigma$ on which there are three distinct principal curvatures is open. Let $\xi$ be a local field of unit normals near $x \in \Sigma$. By Proposition 5.3, $J \xi$ is principal near $x$ with corresponding locally constant principal curvature $\mu=$ $2 \cot 2 r, 0<r<\pi / 2$. Locally, there are two focal maps $\phi_{r}$ and $\phi_{r-\pi / 2}$ arising from $\mu$, and one can compute the rank of each by Proposition 2.1. Let $j$ be the 
maximum rank attained by either of the two focal maps on $\Sigma$. The set $\Omega \subset \Sigma$ on which at least one of the focal maps has rank $j$ is then open in $M$. Let $x \in \Omega$ and choose the local field of normals so that $\phi_{r}$ has rank $j$ near $x$. By Theorem 1 , there is a neighborhood $U$ of $x$ such that $N=\phi_{r} U$ is an embedded complex submanifold, and $U$ lies on the tube of radius $r$ over $N$. By examining Proposition 3.1, we see that there are precisely two ways in which there could be three distinct principal curvatures at each point of $U$. First $N$ could have complex codimension 1 with $A_{\eta}$ having exactly two eigenvalues for each normal $\eta$ to $N$. By a result of Nomizu and Smyth [13, p. 58], $N$ is an open subset of a complex quadric $Q^{m-1}$.

The second possibility is that $N$ is an open subset of a totally geodesic $C P^{k}$, $0<k<m-1$. In either case, the three principal curvatures are constant on $U$, and hence they are locally constant on $\Omega$. Now, as in the proof of Theorem 3, one shows that $\Omega$ is also closed, and so there are three constant principal curvatures on $M$. Another connectedness argument shows that the focal map $\phi_{r}$ maps $M$ into a subset of a particular $Q^{m-1}$ or $C P^{k}$, as the case may be. Finally, a direct calculation shows that among such tubes, only those listed in (b) and (c) actually satisfy the requirement on the Ricci tensor.

Since the three hypersurfaces in Theorem 4 are not Einstein, we have

COROLlaRy 5.5. There are no Einstein real hypersurfaces in $\mathrm{CP}^{\mathrm{m}}$.

\section{REFERENCES}

1. W. Boothby, An introduction to differentiable manifolds and Riemannian geometry, Academic Press, New York, 1975.

2. T. Cecil, Taut immersions of non-compact surfaces into a Euclidean 3-space, J. Differential Geom. 11 (1976), 451-459.

3. Geometric applications of critical point theory to submanifolds of complex projective space, Nagoya Math. J. 55 (1974), 5-31.

4. T. Cecil and P. Ryan, Focal sets of submanifolds, Pacific J. Math. 78 (1978), 27-39.

5. __ Focal sets, taut embeddings and the cyclides of Dupin, Math. Ann. 236 (1978), 177-190.

6. B.-Y. Chen, Extrinsic spheres in Riemannian manifolds, Houston J. Math. 5 (1979), 319-324.

7. B.-Y. Chen and K. Ogiue, Two theorems on Kaehler manifolds, Michigan Math. J. 21 (1974), 225-229.

8. A. Fialkow, Hypersurfaces of a space of constant curvature, Ann. of Math. (2) 39 (1938), 762-785.

9. S. Kobayashi and K. Nomizu, Foundations of differential geometry. Vols. 1, 2, Interscience, New York, 1963, 1969.

10. M. Kon, Pseudo-Einstein real hypersurfaces in complex space forms, J. Differential Geom. 14 (1979), 339-354.

11. Y. Maeda, On real hypersurfaces of a complex projective space, J. Math. Soc. Japan 28 (1976), $529-540$.

12. K. Nomizu, Elie Cartan's work on isoparametric families of hypersurfaces, Proc. Sympos. Pure Math., vol. 27, Part I, Amer. Math. Soc., Providence, R. I., 1974, pp. 191-200.

13. K. Nomizu and B. Smyth, Differential geometry of complex hypersurfaces. II, J. Math. Soc. Japan 20 (1968), 498-521.

14. B. O'Neill, The fundamental equations of a submersion, Michigan Math. J. 13 (1966), 459-469.

15. R. Palais, A global formulation of the Lie theory of transformation groups, Mem. Amer. Math. Soc. No. 22 (1957).

16. H. Reckziegel, On the eigenvalues of the shape operator of an isometric immersion into a space of constant curvature, Math. Ann. 243 (1979), 71-82.

17. P. Ryan, Hypersurfaces with parallel Ricci tensor, Osaka J. Math. 8 (1971), 251-259.

18. , Homogeneity and some curvature conditions for hypersurfaces, Tôhoku Math. J. 21 (1969), $363-388$. 
19. B. Smyth, Differential geometry of complex hypersurfaces, Ann. of Math. (2) 85 (1967), 246-266.

20. R. Takagi, Real hypersurfaces in a complex projective space with constant principal curvatures, J. Math. Soc. Japan 27 (1975), 43-53.

21. __ Real hypersurfaces in a complex projective space with constant principal curvatures. II, J. Math. Soc. Japan 27 (1975), 507-516.

22. Y. Tashiro and S. Tachibana, On Fubinian and C-Fubinian manifolds, Kōdai Math. Sem. Rep. 15 (1963), 176-183.

23. T. Thomas, Extract from a letter by E. Cartan concerning my note: On closed spaces of constant mean curvature, Amer. J. Math. 59 (1937), 793-794.

24. A. Weinstein, Distance spheres in complex projective spaces, Proc. Amer. Math. Soc. 39 (1973), 649-650.

Department of Mathematics, College of the Holy Cross, Worcester, Massachusetts 01610

Department of Mathematics, Indiana University at South Bend, South Bend, Indiana 46615 